\title{
The Brain Thermal Response as a Potential Neuroimaging Biomarker of Cerebrovascular Impairment
}

\author{
(D)C.C. Fleischer, (D). Wu, (DD. Qiu, (D) S.-E. Park, (D) F. Nahab, and (D) S. Dehkharghani
}

\begin{abstract}
BACKGROUND AND PURPOSE: Brain temperature is critical for homeostasis, relating intimately to cerebral perfusion and metabolism. Cerebral thermometry is historically challenged by the cost and invasiveness of clinical and laboratory methodologies. We propose the use of noninvasive MR thermometry in patients with cerebrovascular disease, hypothesizing the presence of a measurable brain thermal response reflecting the tissue hemodynamic state.
\end{abstract}

MATERIALS AND METHODS: Contemporaneous imaging and MR thermometry were performed in 10 patients (32-68 years of age) undergoing acetazolamide challenge for chronic, anterior circulation steno-occlusive disease. Cerebrovascular reactivity was calculated with blood oxygen level-dependent imaging and arterial spin-labeling methods. Brain temperature was calculated pre- and post-acetazolamide using previously established chemical shift thermometry. Mixed-effects models of the voxelwise relationships between the brain thermal response and cerebrovascular reactivity were computed, and the significance of model coefficients was determined with an $F$ test $(P<.05)$.

RESULTS: We observed significant, voxelwise quadratic relationships between cerebrovascular reactivity from blood oxygen leveldependent imaging and the brain thermal response ( $x$ coefficient $=0.052, P<.001 ; x^{2}$ coefficient $=0.0068, P<.001$ ) and baseline brain temperatures $\left(x\right.$ coefficient $=0.59, P=.008 ; x^{2}$ coefficient $=-0.13, P<.001$ ). A significant linear relationship was observed for the brain thermal response with cerebrovascular reactivity from arterial spin-labeling $(P=.001)$.

CONCLUSIONS: The findings support the presence of a brain thermal response exhibiting complex but significant interactions with tissue hemodynamics, which we posit to reflect a relative balance of heat-producing versus heat-dissipating tissue states. The brain thermal response is a potential noninvasive biomarker for cerebrovascular impairment.

ABBREVIATIONS: $\mathrm{ACZ}=$ acetazolamide; $\mathrm{ASL}=$ arterial spin-labeling; $\mathrm{BOLD}=$ blood oxygen level-dependent; $\mathrm{BTR}=$ brain thermal response; $\mathrm{CMRO}_{2}=$ cerebral metabolic rate of oxygen; $\mathrm{CVR}=$ cerebrovascular reactivity; $\mathrm{MRSI}=\mathrm{MR}$ spectroscopic imaging; $\mathrm{OEF}=$ oxygen extraction fraction

C hronic cerebrovascular disease remains a common cause of death and disability. While treatment advances have reduced stroke rates in such individuals, there exists a subset of patients at heightened ongoing risk linked to a tenuous state of so-called misery perfusion and characterized by exhaustion of hemodynamic compensatory mechanisms. ${ }^{1-4}$ Although individual pa-

Received January 17, 2017; accepted after revision July 9.

From the Department of Biomedical Engineering (C.C.F., S.-E.P.), Emory University and Georgia Institute of Technology, Atlanta, Georgia; and the Departments of Radiology and Imaging Sciences (C.C.F., J.W., D.Q., S.D.), Neurology (F.N., S.D.), and Pediatrics (F.N.), Emory University School of Medicine, Atlanta, Georgia.

Dr Dehkharghani is currently affiliated with the Department of Radiology, Neuroradiology Section, New York University, New York, New York.

Deqiang Qiu is supported by National Institutes of Health grants AG25688, AG42127, AG49752, and AG51633.

Abstract previously presented at: Annual Meeting and Exhibition of the International Society for Magnetic Resonance in Medicine, May 7-13, 2016; Singapore; and the Annual Meeting of the American Society of Neuroradiology, May 21-26, 2016; Washington, DC tients may deviate from the proposed paradigm of decompensation, misery perfusion confers an increased risk for future ischemic injury, and refined characterization of the pathophysiologic underpinnings may allow improved prognostication or therapy selection in such patients. ${ }^{1,2}$

Historically, ${ }^{15} \mathrm{O}$-PET has been used to identify misery perfusion as defined by disturbances in $\mathrm{CBF}, \mathrm{CBV}$, oxygen extraction fraction $(\mathrm{OEF})$, and the cerebral metabolic rate of oxygen $\left(\mathrm{CMRO}_{2}\right) \cdot{ }^{5-7}$ Challenges inherent to ${ }^{15} \mathrm{O}$-PET, including reliance on short half-life radioisotope tracers and radiation exposure, compel the development of pragmatic, ideally noninvasive and noncontrast, MR imaging biomarkers to identify and characterize misery perfusion. Such approaches would enable safe and repeat-

Please address correspondence to Seena Dehkharghani, MD, Department of Radiology, Neuroradiology Section, New York University, 660 First Ave, New York, New York 10016; e-mail: seena.dehkharghani@nyumc.org

- Indicates open access to non-subscribers at www.ajnr.org

http://dx.doi.org/10.3174/ajnr.A5380 
able imaging, allowing structural and functional characterization of the tissue state. ${ }^{8}$ Among MR imaging techniques, cerebrovascular reactivity (CVR) measured from blood oxygen level-dependent (BOLD) imaging or calculated from arterial spin-labeling (ASL) has been proposed using hemodynamic augmentation such as induced hypercapnia or acetazolamide (ACZ) administration. ${ }^{8-10}$ Impaired CVR is strongly associated with ischemic risk in patients with carotid steno-occlusive disease, suggesting that early identification combined with appropriate intervention may improve outcomes. ${ }^{11,12}$

Under-represented in such studies is the role of brain temperature, a homeostatic variable inextricably linked to CBF and cerebral metabolism. ${ }^{13,14}$ Increased brain and systemic temperatures strongly potentiate ischemic injury due to the high sensitivity of neuronal substrate to even small temperature fluctuations. ${ }^{15-19}$ Cerebral thermoregulation remains poorly understood, owing to historically limited methodologies consisting of highly invasive and costly implantable probes. Past study has thus relied primarily on animal models or used systemic temperature as a surrogate for brain temperature, with limited empirical exploration of the effect of hemodynamic impairment on thermal dynamics. ${ }^{15,17,20-22}$

Our goal was to explore the feasibility of a "brain thermal response" (BTR) as a potential MR neuroimaging biomarker for hemodynamic impairment by characterizing the relationship between BTR and CVR. We propose BTR as a measurement of dynamic brain temperature fluctuations in response to a hemodynamic maneuver, hypothesizing the presence of detectable, coherent temperature response differences paralleling tissue-level hemodynamic impairment.

\section{MATERIALS AND METHODS Experimental Design}

Retrospective analysis was performed on data acquired in patients undergoing treatment for chronic, unilateral anterior circulation steno-occlusive disease and recurring neurovascular events (minor stroke or transient ischemic attack), referred by a vascular neurologist to undergo a standardized institutional protocol in our chronic neurovascular disease patient population. A waiver of Health Insurance Portability and Accountability Act authorization and informed consent was approved by the institutional review board. Exclusion criteria included contraindications to MR imaging, renal impairment, sensitivity to sulfa derivatives, extreme head motion during the scan, or inability to comply with the study protocol for 2-day multiphasic MR imaging (3 subjects excluded). After exclusion, 10 subjects between 32 and 68 years of age (mean, $46 \pm 13$ years) were included in the study ( 8 women, 32-68 years of age; mean, $41 \pm 12$ years; 2 men, 56 and 61 years of age).

\section{MR Imaging and Spectroscopy}

Subjects completed a standardized, 2-day MR imaging protocol with ACZ challenge (Fig 1). Multivoxel MR spectroscopic imaging (MRSI), ASL, and BOLD data were acquired both pre- and post-ACZ. All scanning was performed on a Tim Trio 3T wholebody MR imaging scanner (Siemens, Erlangen, Germany) with a 32-channel head-array coil. A 3D T1-weighted MPRAGE (TR/ $\mathrm{TE}=1900 / 3.5 \mathrm{~ms}, \mathrm{~T} 1=900 \mathrm{~ms}$, flip angle $=9^{\circ}, 1 \mathrm{~mm}$ isotropic resolution) anatomic image was used to guide MRSI grid place-

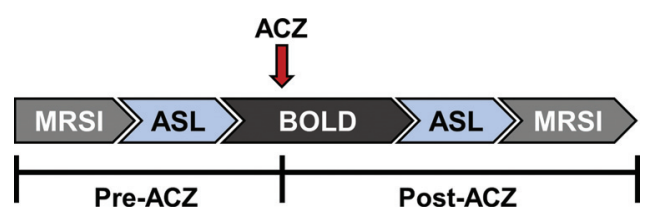

FIG 1. Schematic of MR imaging scan protocol. ACZ was injected 5 minutes after the start of the BOLD sequence and data were acquired for $\sim 10$ minutes post-injection. ASL and MRSI were acquired pre- and post-ACZ.

ment and to plan the ASL and BOLD experiments. A 6-minute multi-TI ASL sequence with background suppression was acquired with a multidelay single-shot 3D gradient- and spin-echo readout and flow-sensitive alternating inversion recovery tagging with the following parameters: TR/TE $=3600 / 19.3 \mathrm{~ms}, \mathrm{FOV}=$ $320 \times 160 \mathrm{~mm}$, section thickness $=3.5 \mathrm{~mm}, 32$ sections, 5 averages, and 10 postlabel delays: first TI $=300 \mathrm{~ms}$, increment $=300$ ms. A 20-minute continuous BOLD study was performed, beginning with 5 minutes of baseline acquisition, after which ACZ (Diamox, $1 \mathrm{~g}$ dissolved in $10 \mathrm{~mL}$ of normal saline) was administered without scan interruption or patient repositioning. ACZ was delivered via slow, manual IV infusion for 3-5 minutes in aliquots of approximately $2 \mathrm{~mL}$ per minute, followed by a normal saline flush, after which the remaining $\sim 10$ minutes of continuous and uninterrupted BOLD imaging was performed. BOLD data were acquired with a gradient-echo echo-planar imaging sequence $(\mathrm{TR} / \mathrm{TE}=2000 / 30 \mathrm{~ms}, \mathrm{FOV}=220 \mathrm{~mm}$, matrix $=64 \times 64$, section thickness $=4 \mathrm{~mm}, 30$ sections).

MRSI scans for thermometry were acquired pre- and postACZ injection with the semi-localized by adiabatic selective refocusing sequence ${ }^{23-25}$ with an $8 \times 8$ voxel ROI with the center of the grid aligned over the midline of the brain $(\mathrm{TR} / \mathrm{TE}=1700 / 35$ $\mathrm{ms}, 3$ averages, $10 \times 10 \times 15 \mathrm{~mm}^{3}$ nominal voxel size, $16 \times 16$ voxel acquisition matrix with lipid saturation outside the ROI, 50-Hz water-suppression bandwidth).

For each subject, the diseased hemisphere was identified on contemporaneous 3D time-of-flight brain MRA, as well as on conventional DSA or CTA when available. All imaging was evaluated by a dedicated neuroradiologist with subspecialty certification and 8 years of experience in advanced stroke imaging (S.D.), and a vascular neurologist with $>10$ years of experience in stroke and neurovascular imaging (F.N.).

\section{Cerebrovascular Reactivity}

CVR pre- versus post-ACZ administration was calculated using both relative $\mathrm{CBF}$ from ASL data $\left(\mathrm{CVR}_{\mathrm{ASL}}\right)$ and $\mathrm{BOLD}$ signal augmentation $\left(\mathrm{CVR}_{\mathrm{BOLD}}\right)$. For $\mathrm{CVR}_{\mathrm{ASL}}$, the $\mathrm{CBF}$ map from the post-ACZ ASL acquisition $\left(\mathrm{CBF}_{\text {post }}\right)$ was coregistered to the pre$\mathrm{ACZ} \mathrm{CBF}\left(\mathrm{CBF}_{\text {pre }}\right)$ map to correct for motion effects and smoothed with a Gaussian kernel (full width at half maximum $=$ $8 \mathrm{~mm}$ ). The raw data were fit with a kinetic model reported elsewhere using the inline vendor-supplied processing output. ${ }^{26,27}$ $\mathrm{CVR}_{\mathrm{ASL}}$ was calculated from Equation 1.

1)

$$
\mathrm{CVR}_{\mathrm{ASL}}=\frac{\mathrm{CBF}_{\text {post }}-\mathrm{CBF}_{\text {pre }}}{\mathrm{CBF}_{\text {pre }}} \times 100 \%
$$

$\mathrm{CVR}_{\mathrm{BOLD}}$ was calculated from BOLD images following realignment to correct for motion and smoothed with a Gaussian kernel 
(full width at half maximum $=8 \mathrm{~mm}$ ). The voxelwise mean intensity values from 30 BOLD volumes acquired before ACZ injection $\left(\mathrm{BOLD}_{\text {pre }}\right)$ and the mean intensity values from the final 30 BOLD volumes $\left(\mathrm{BOLD}_{\text {post }}\right)$ were calculated. $\mathrm{CVR}_{\mathrm{BOLD}}$ was then computed with Equation 2.

$$
\mathrm{CVR}_{\mathrm{BOLD}}=\frac{\mathrm{BOLD}_{\text {post }}-\mathrm{BOLD}_{\text {pre }}}{\mathrm{BOLD}_{\text {pre }}} \times 100 \%
$$

The final CVR maps were coregistered to the T1-weighted images in the patient coordinate system, to facilitate voxelwise analysis with the MRSI data, and were resampled to a nominal $1 \mathrm{~mm}$ isotropic resolution. For $\mathrm{CVR}_{\mathrm{ASL}}$ values, augmentation of $<30 \%$ was used as a threshold to identify regions of hemodynamic impairment as previously reported. ${ }^{10,28-30}$ For $\mathrm{CVR}_{\mathrm{BOLD}}, 70 \%$ of the subject-wise mean contralateral CVR value was used as a threshold. The use of an individual cutoff value was chosen to minimize variation from intersubject BOLD fluctuations. To generate the CVR maps for display, a brain mask was generated using the T1weighted image with the FSL Brain Extraction Tool (http://fsl. fmrib.ox.ac.uk/fsl/fslwiki/BET), ${ }^{31}$ and masked CVR color maps were generated in Matlab (Version 2015a; MathWorks, Natick, Massachusetts).

\section{Thermometry and Brain Thermal Response}

MRSI data for thermometry were processed using LCModel (http://www.lcmodel.com) $)^{32}$ as reported previously. ${ }^{19,24,25}$ Temperature analysis and localization of the grid onto CVR maps were performed using in-house software written in Matlab. Specifically, absolute temperature was calculated using the difference between water and NAA proton resonance frequencies and the relationship $-0.01 \mathrm{ppm} /{ }^{\circ} \mathrm{C} .{ }^{33,34}$ Voxelwise temperature changes were calculated as a difference between post- and pre-ACZ absolute temperatures $\left(\mathrm{T}_{\text {post }}-\mathrm{T}_{\text {pre }}\right)$. The unitless parameter, BTR, was defined as the temperature change normalized to the preACZ temperature (Equation 3). Temperature maps were calculated on a voxelwise basis and interpolated using bicubic interpolation for visualization purposes.

$$
\mathrm{BTR}=\frac{\mathrm{T}_{\text {post }}-\mathrm{T}_{\text {pre }}}{\mathrm{T}_{\text {pre }}}
$$

To facilitate the voxelwise analysis between BTR and CVR, the MRSI grid was localized onto the CVR images. Because the MRSI grid was planned using the T1-weighted image in the patient coordinate system, the data for both MRSI and CVR use the same coordinate system. The MRSI grid was first rotated parallel to the axial plane of the patient coordinate system, followed by a linear translation of the center of the MRSI grid to the origin $(0,0,0 \mathrm{~mm})$. These same transformations were also applied to the CVR data, which were then resliced using the trilinear interpolation method while retaining the nominal $1-\mathrm{mm}$ isotropic resolution. The same transformation and reslicing were also applied to the T1-weighted image to visually confirm the location of the MRSI grid onto the CVR map and to overlay the temperature grid onto the anatomic image. The final CVR data were interpolated to an $8 \times 8$ grid $\left(10 \times 10 \times 15 \mathrm{~mm}^{3}\right.$ voxel resolution), identical to the MRSI grid, and an average CVR value was calculated for each MRSI voxel.

\section{Statistical Analysis}

Statistical analysis was performed with SPSS (Version 22.0; IBM, Armonk, New York). Hemispheric comparisons (ipsilateral versus contralateral) of baseline brain temperature and BTR were performed for both random and fixed effects. However, most of the analysis was performed voxelwise to permit comparison of BTR, baseline brain temperature, and CVR and to explore localized impairment across the entire ROI. The voxelwise relationships among these parameters were determined with a mixedeffects model (Equation 4), in which $\vec{y}$ is the vector of measured BTR or baseline temperature values; $\hat{X}$ is a matrix of fixed effects (CVR, square of CVR, and intercept) and the corresponding vector of fixed-effect coefficients, $\vec{\beta}$; $\hat{Z}$ is a matrix of random between-subject variations and the corresponding vector of random-effect coefficients, $\vec{b}$; and $\vec{\varepsilon}$ is a vector of random errors.

$$
\vec{y}=\hat{X} \vec{\beta}+\hat{Z} \vec{b}+\vec{\varepsilon}
$$

The restricted maximum likelihood estimation with 150 iterations was used to fit the model using either unstructured or firstorder autoregressive covariance structures, along with a random intercept to account for multiple nonindependent measurements from voxels within each subject. The best fit models, comparing both linear and quadratic models along with different covariance structures for the random effects, were determined with the Schwarz Bayesian information criterion and a value of $>10$ to establish differences among models. ${ }^{35}$ In the case in which the Schwarz Bayesian information criterion was $<10$, parsimony was applied for the final model selection. $d f$ for the mixed-models were determined with the Satterthwaite approximation. Exponential models were also explored but did not converge. The significance of the regression was determined with the mixed-model $F$ statistic and $P<.05$.

\section{RESULTS}

Patient characteristics are presented in Table 1. Representative $\mathrm{CVR}_{\mathrm{BOLD}}$ and $\mathrm{CVR}_{\mathrm{ASL}}$ augmentation maps, the corresponding BTR map, and a diffusion-weighted image for a patient with unilateral left middle cerebral artery stenosis are presented in Fig 2. The position of the MRSI grid is shown in white.

Best-fit models and respective parameters for the voxelwise relationships are summarized in Table 2. We observed a significant, positive quadratic relationship for all voxels between BTR and $\mathrm{CVR}_{\mathrm{BOLD}}$ (Fig 3; CVR coefficient, $P<.001$; $\mathrm{CVR}^{2}$ coefficient, $P<.001)$. A significant, negative quadratic relationship between baseline brain temperature and $\mathrm{CVR}_{\mathrm{BOLD}}$ was also observed (CVR coefficient, $P=.008$; $\mathrm{CVR}^{2}$ coefficient, $P<.001$ ). No significant global relationships were observed with $\mathrm{CVR}_{\mathrm{ASL}}$.

In the analysis of $\mathrm{CVR}_{\mathrm{ASL}}$, thresholded and dichotomized into voxels exhibiting either $<30 \%$ augmentation (impaired) or $\geq 30 \%$ augmentation (healthy), a significant linear relationship was observed for BTR with $\mathrm{CVR}_{\mathrm{ASL}}$ of $\geq 30 \%$ augmentation $(P=$ $.001)$. No significant relationships were observed for the thresholded $\mathrm{CVR}_{\mathrm{ASL}}$ values with baseline brain temperature. Similarly, we used the $\mathrm{CVR}_{\mathrm{BOLD}}$ values thresholded subject-wise at $<70 \%$ (impaired) or $\geq 70 \%$ (healthy) of the mean contralateral CVR augmentation. Significant relationships were observed between 
BTR and $\mathrm{CVR}_{\mathrm{BOLD}}$ of $<70 \%$ (positive quadratic: CVR coefficient, $P<.001$; $\mathrm{CVR}^{2}$, coefficient, $P<.001$ ) and $\geq 70 \%$ (linear, $P=.02$ ) of the mean contralateral hemisphere. Additionally, significant relationships for baseline brain temperature and $\mathrm{CVR}_{\mathrm{BOLD}}$ of $<70 \%$ (negative quadratic: CVR, $P<.001$; $\mathrm{CVR}^{2}$,

Table 1: Patient characteristics

\begin{tabular}{lccl}
\hline $\begin{array}{c}\text { Patient } \\
\text { No. }\end{array}$ & $\begin{array}{c}\text { Age } \\
\text { (years) }\end{array}$ & Sex & \multicolumn{1}{c}{ Presentation and Diagnosis } \\
\hline 1 & 41 & F & Left-sided-predominate supraclinoid ICA stenosis \\
2 & 61 & M & Left cervical ICA stenosis; aphasia \\
3 & 39 & F & Right-sided-predominate ICA stenosis; recurrent minor stroke \\
4 & 32 & F & Left M1 stenosis; left monocular vision loss and recurrent right hemiparesis \\
5 & 56 & M & Intracranial ICA stenosis; recurrent TIA \\
6 & 68 & F & Left ICA stenosis; recurrent left hemispheric TIA and borderzone ischemia \\
7 & 33 & F & Right-sided-predominate supraclinoid ICA stenosis; Moyamoya disease \\
& & & following STA-MCA bypass and recurrent TIA \\
8 & 36 & F & Left Ml stenosis; recurrent left deep borderzone ischemia \\
9 & 38 & F & Right-sided-predominate intracranial stenosis; recurrent MCA infarctions \\
10 & 51 & F & Left ICA stenosis; recurrent TIA \\
\hline
\end{tabular}

Note:-STA indicates superficial temporal artery.
$P<.001$ ) and $\geq 70 \%$ (linear, $P=.001$ ) of the contralateral hemisphere were also observed.

We did not observe a significant difference in BTR $(P=.58)$ or baseline temperature ( $P=.38$ ) between the ipsilateral and contralateral hemispheres. However, within-subject variations (random-effects, Equation 4) were highly significant for both BTR $(P<.001)$ and baseline brain temperature $(P<.001)$.

\section{DISCUSSION}

The results support the presence of a BTR as a potential neuroimaging biomarker reflecting a hypothesized, convolved interaction of hemodynamic and metabolic tissue properties. The measurement of a BTR is feasible in routine clinical MR imaging implementations,

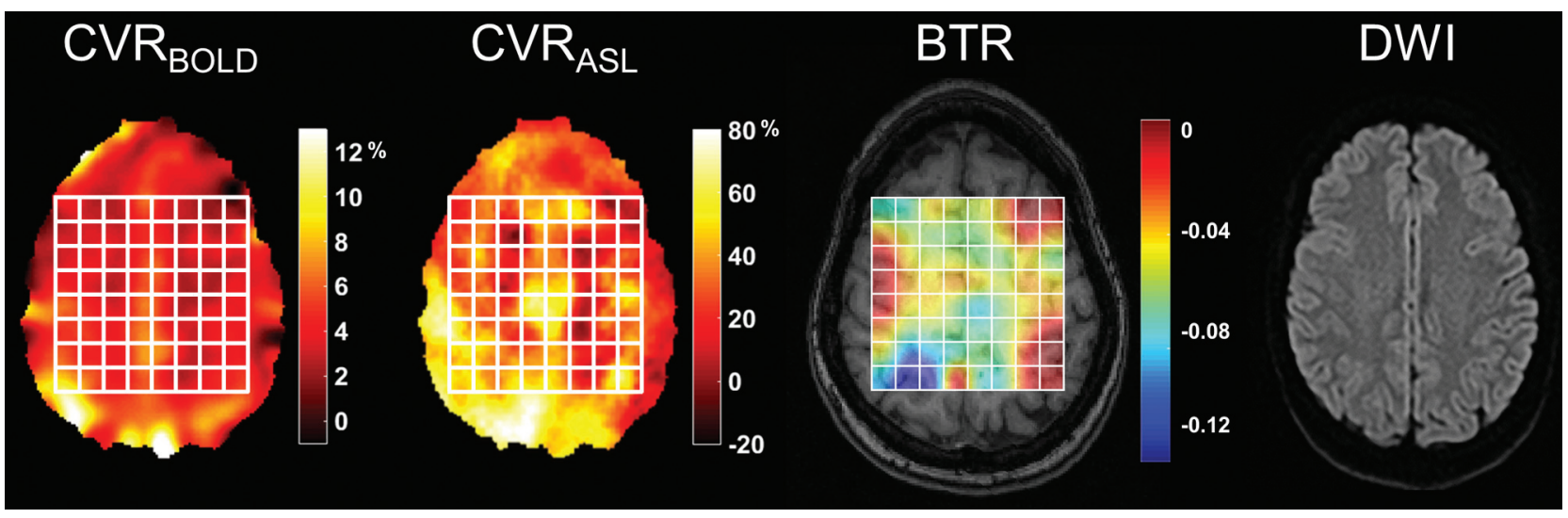

FIG 2. Representative CVR percentage augmentation maps calculated with BOLD and ASL, along with a BTR map overlaid on a T7-weighted image. Images are all from the same subject (a 32-year-old woman) with unilateral left MCA stenosis without infarction. The white grid overlay represents the MR thermometry grid derived from multivoxel spectroscopy analysis using the water-NAA chemical shift difference. Diffusionweighted imaging demonstrates no evidence for acute infarction. Images are displayed in the radiologic convention. Impaired CVR in the left hemisphere is present in both BOLD and ASL, with a greater severity of impairment in ASL likely related to delay sensitivity and tag decay (see text). The BTR map demonstrates an asymmetric thermal response, with less brain cooling following vasodilatory stimulus in the diseased left hemisphere, indicated by lower and more positive BTR values and corresponding primarily to the areas of greatest impairment in the anterior and posterior MCA borderzone territories.

Table 2: Parameter estimates calculated with a mixed-effects model of BTR and baseline brain temperature as a function of CVR

\begin{tabular}{|c|c|c|c|c|c|c|c|c|c|c|c|c|}
\hline & \multicolumn{4}{|c|}{ CVR } & \multicolumn{4}{|c|}{$\mathrm{CVR}^{2}$} & \multicolumn{4}{|c|}{ Intercept } \\
\hline & Coefficient & $F$ & $d f^{a}$ & $P$ Value & Coefficient & $\boldsymbol{F}$ & $d f^{a}$ & $P$ Value & Coefficient & $F$ & $d f^{\mathrm{a}}$ & $P$ Value $^{\mathrm{b}}$ \\
\hline \multicolumn{13}{|l|}{ BTR } \\
\hline CVR & -0.052 & 74.1 & 1,513 & $<.001$ & 0.0068 & 57.5 & 1,619 & $<.001$ & 0.031 & 6.0 & 1,35 & .02 \\
\hline $\mathrm{CVR}_{\mathrm{ASL}}$ & 0.0018 & 1.2 & $1,8.0$ & .30 & - & - & - & - & -0.069 & 205 & $1,3.8$ & $<.001$ \\
\hline $\mathrm{CVR}_{\mathrm{BOLD}}<70^{\circ}$ & -0.096 & 59.7 & 1,97 & $<.001$ & 0.024 & 25.0 & 1,128 & $<.001$ & 0.031 & 2.8 & 1,11 & .12 \\
\hline $\mathrm{CVR}_{\mathrm{BOLD}} \geq 70^{\circ}$ & 0.0070 & 5.5 & 1,200 & .02 & - & - & - & - & -0.081 & 46.0 & 1,51 & $<.001$ \\
\hline $\mathrm{CVR}_{\mathrm{ASL}}<30^{\mathrm{d}}$ & 0.0018 & 1.1 & $1,7.4$ & .33 & - & - & - & - & -0.068 & 183.2 & 1,69 & $<.001$ \\
\hline$C V R_{A S L} \geq 30^{d}$ & 0.0014 & 12.1 & 1,47 & .001 & - & - & - & - & -0.10 & 33.5 & 1,44 & $<.001$ \\
\hline \multicolumn{13}{|l|}{$\mathrm{T}_{\text {pre }}{ }^{\mathrm{e}}\left({ }^{\circ} \mathrm{C}\right)$} \\
\hline CVR $\mathrm{BOLD}_{\mathrm{BO}}$ & 0.59 & 7.0 & 1,598 & .008 & -0.13 & 14.8 & 1,630 & $<.001$ & 37.5 & 4871 & 1,27 & $<.001$ \\
\hline $\mathrm{CVR}_{\mathrm{ASL}}$ & -0.039 & 0.9 & $1,7.3$ & .39 & - & - & - & - & 38.4 & 8655 & $1,6.0$ & $<.001$ \\
\hline $\mathrm{CVR}_{\mathrm{BOLD}}<70^{\circ}$ & 2.1 & 19.5 & 1,139 & $<.001$ & -0.65 & 12.9 & 1,150 & $<.001$ & 37.1 & 1810 & 1,17 & $<.001$ \\
\hline $\mathrm{CVR}_{\mathrm{BOLD}} \geq 70^{\circ}$ & -0.38 & 10.7 & 1,429 & .001 & - & - & - & - & 39.0 & 4575 & 1,28 & $<.001$ \\
\hline $\mathrm{CVR}_{\mathrm{ASL}}<30^{\mathrm{d}}$ & -0.049 & 1.1 & $1,6.8$ & .34 & - & - & - & - & 38.5 & 12,953 & $1,6.5$ & $<.001$ \\
\hline $\mathrm{CVR}_{\mathrm{ASL}} \geq 30^{\mathrm{d}}$ & 0.0026 & 0.01 & 1,198 & .91 & - & - & - & - & 37.5 & 1094 & 1,27 & $<.001$ \\
\hline
\end{tabular}

${ }^{a} d f$ calculated with the Satterthwaite approximation are reported as numerator, denominator.

${ }^{b} P$ value represents the significance of a nonzero intercept.

${ }^{\mathrm{C}} \mathrm{CVR}_{\mathrm{BOLD}}$ values thresholded to $70 \%$ of the subject-wise mean contralateral value.

${ }^{d} C_{\text {CVR }}$ ASL values thresholded to $30 \%$ augmentation.

${ }^{\mathrm{e}} \mathrm{T}_{\mathrm{pre}}$ is the baseline brain temperature. 


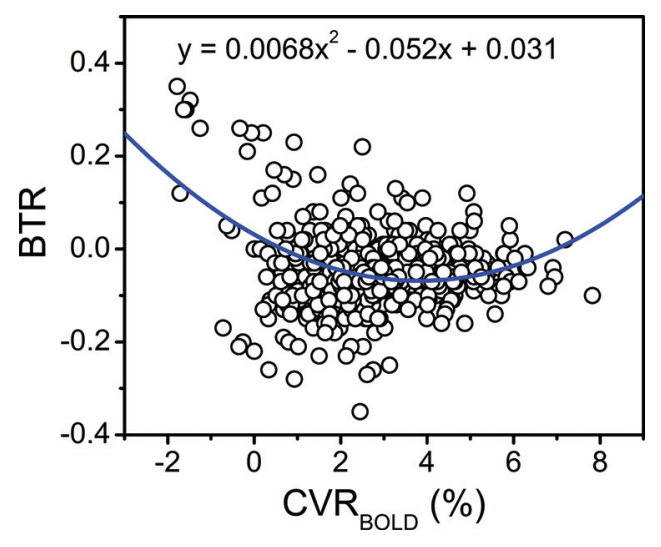

FIG 3. Voxelwise relationship of the BTR versus BOLD MR imaging signal augmentation following $A C Z$ administration. The blue line and corresponding equation represent the fit of the raw data (circles) calculated with a mixed-effects model. A significant quadratic relationship between BTR and $C V R_{\mathrm{BOLD}}$ was identified for all voxels, wherein an initially negative slope is observed at low $C V R_{B O L D}$ but a positive trend is observed for $\mathrm{CVR}_{\mathrm{BOLD}}$ augmentation upward of approximately $4 \%$ (see "Results" and "Discussion"; $F, x^{2}=57.5, P<.001$; $F, x=74.1, P<.001)$.

with significant relationships identified between both BTR and baseline brain temperature relative to CVR, supporting further exploration of BTR as a fully noninvasive and dynamic biomarker. The use of voxelwise measurements facilitated a nuanced description of local hemodynamics and temperature, allowing contemporaneous, multiparametric classification of the diseased hemisphere, all potentially achievable in a single imaging session. MR chemical shift thermometry, exploiting the temperature-dependent difference between water and the methyl proton resonance of NAA, was chosen to approximate temperature using our previously reported protocol for phantom as well as human and nonhuman primate in vivo thermometry. ${ }^{19,24,25,33}$ The present study takes advantage of the unique pathophysiologic attributes of chronic cerebrovascular ischemia, in a cohort allowing multiphasic interrogation of a flow-temperature relationship, which may not be pragmatic in the clinical acute stroke setting. Ishigaki et $\mathrm{al}^{36}$ previously reported the use of PET combined with localized, single-voxel MR spectroscopy-based thermometry to explore the relationship of brain temperature in steno-occlusive disease, observing positive correlations between the brain temperature difference (ipsilateral-contralateral hemisphere) with both CBV and OEF. A limited number of additional studies using single-voxel MR spectroscopy thermometry have observed higher temperatures in ischemic brain tissues, further supporting the investigation of brain temperature in the development of a pathophysiologic framework defining cerebral energetics. ${ }^{16,36-38}$

Our analysis of the relationship between BTR and baseline brain temperature with CVR was performed voxelwise to examine whole-brain effects in patients with steno-occlusive disease. CVR and flow augmentation are generally hypothesized to reduce brain temperature and might, therefore, have been expected to produce a negative BTR. Our results were, however, more complex, and the observed flow-temperature relationship may not be immediately intuitive but, on deeper examination, can be reconciled with prevailing theories regarding cerebral blood flow, temperature, oxygen metabolism, and BOLD signal evolution. Exist- ing models of cerebral temperature homeostasis propose a dynamic modulation of heat-producing mechanisms (oxygen cleavage from hemoglobin, glycolysis, and generation of metabolic by-products) and heat dissipation through the inflow of cooler systemic blood with lesser contributions from conduction to the brain surface. This is predicated on the notion of heat sink/ radiator effects of inflowing blood, cooling tissues through the removal of metabolic waste.

We would also anticipate relative heating among those tissues with insufficient blood flow to meet metabolic demands. Conceptually, a linear or at least monotonically changing thermal response as a function of flow might be expected. However, several factors could interfere with this conceptual framework: 1) the temperature-flow relationship may vary radially within the brain, whereby superficial structures closer to the surface CSF are cooler than arterial inflow, thus warming with increasing flow; and 2) oxygen extraction, a primary contributor to the production of thermal waste, may be variably upregulated depending on the depth of misery perfusion, ostensibly at its highest among tissues with the most impaired flow. However, as demonstrated in several past studies, the capacity for flow augmentation may remain even among tissues with OEF elevation. The downregulation of OEF among such tissues during hemodynamic augmentation may occur on a time scale shorter than the duration of the BOLD scan and therefore shorter than the separation in time between CBF or thermometry imaging. This may, in part, drive a component of time shifts observed in BOLD signal augmentation if, for instance, a very high OEF must be overcome before BOLD augmentation can be detectable (ie, for deoxyhemoglobin dilution to occur sufficiently). ${ }^{39}$ Accordingly, CVR augmentation following ACZ was initially anticipated to produce negative BTR values as a result of the increased inflow of cooler arterial blood, acting as a heat sink for warmer cerebral temperatures and removing metabolic byproducts, including thermal waste.

Such interactions could underlie the observed quadratic relationship between BTR with $\mathrm{CVR}_{\mathrm{BOLD}}$, wherein a negative BTRCVR relationship was present at a low CVR but a positive trend was observed for $\mathrm{CVR}_{\mathrm{BOLD}}$ augmentation upward of approximately $4 \%$. The far left of the curve follows the expected relationship, with decreasing (ie, more negative) BTR accompanied by increasing CVR augmentation and, indirectly, CBF. The source of the observed positive correlation between BTR and CVR above $4 \%$ remains inconclusive within the limitations of this exploratory analysis, in the absence of concurrent $\mathrm{OEF}$ and $\mathrm{CMRO}_{2}$ interrogation (see below).

An opposing relationship (positive-versus-negative quadratic curve) was observed between baseline brain temperature and $\mathrm{CVR}_{\mathrm{BOLD}}$, suggesting that resting brain temperature alone may not fully explain the dynamics of the brain temperature response to a hemodynamic maneuver. This difference may, in part, reflect that baseline brain temperature is inherently a known indicator of diseased tissue energetics, with higher temperature associated positively with hypoperfusion and ischemia. ${ }^{16,19,36-38}$ The benefit of BTR is the ability to quantify a maneuver response, thus producing a form of physiologic contrast with the potential for characterizing not only static tissue impairment, as with baseline brain temperatures, but also the dynamics of the hemodynamic re- 
sponse in a manner similar to that of CVR. The overall quadratic relationships suggest the presence of multiple regimes of temperature regulation or dysregulation across the brain, determined by additional hemodynamic parameters such as OEF and $\mathrm{CMRO}_{2}$.

In addition to the relationships observed among BTR, baseline temperature, and $\mathrm{CVR}_{\mathrm{BOLD}}$ for all voxels, significant associations were also observed for the $\mathrm{CVR}_{\mathrm{BOLD}}$ values thresholded to $70 \%$ of the contralateral hemispheres, demonstrating a robust interaction between BTR and $\mathrm{CVR}_{\mathrm{BOLD}}$. The use of thresholding to explore voxelwise relationships enabled discrimination between impaired and healthy voxels, given the absence of a healthy control cohort undergoing the MR imaging ACZ protocol for comparison in this study.

Using $\mathrm{CVR}_{\mathrm{ASL}}$ to study the same relationships resulted in a single significant correlation for BTR with $\mathrm{CVR}_{\mathrm{ASL}}$ of $\geq 30 \%$. Inherent challenges to ASL perfusion in highly delayed or low-flow environments, such as expected even in compensated territories downstream from steno-occlusive disease, may impact its use in this context, even when using multi-TI approaches to the extent governed by tag decay. We suspect that several, potentially divergent errors in ASL quantitation in such patients may have confounded the BTR relationship with $\mathrm{CVR}_{\mathrm{ASL}}$, among them: 1) exaggerated hypoperfusion if tagged spins are undelivered to the vascular network at the selected TI; 2) exaggerated hyperperfusion if tagged spins are delivered but linger in the arterial network overlying the tissue of interest (particularly when crusher gradients are not used, or when partial volume effects preclude reliable segmentation of the cortical tissues from such overlying vessels, both of which exist in our protocol); or 3) exaggerated hypoperfusion due to excessive tag decay, which may affect our measurements, particularly at the longest TI of $3000 \mathrm{~ms}$. An additional circumstance of negative $\mathrm{CVR}_{\mathrm{ASL}}$, which we have observed to occur despite preserved BOLD and dynamic susceptibility contrast augmentation, exists in tissues with large delays. Arterial transit artifacts at baseline that spuriously elevate perfusion are somewhat cleared following ACZ but appear not to increase the apparent tissue perfusion to the same extent that the arterial transit artifacts previously had, therefore leading to apparently negative CVR. While exploratory, we suspect that temperature dynamics, together with flow, blood volume, and oxygen use, may be better subsumed by $\mathrm{CVR}_{\mathrm{BOLD}}$, albeit in an entangled manner requiring deeper, multiparametric study. Taken together, the observed trends imply the presence of at least 2 distinct regimes for BTR as a function of CVR augmentation: 1) decreasing BTR in response to increasing CVR (negative relationship) as predicted from existing models of brain temperature regulation, which we hypothesize may be dominated by heat-dissipating CBF augmentation over metabolic heat production, with decreasing temperatures in response to $\mathrm{ACZ}^{13,14}$; and 2) a positive BTR-CVR regime in which increasing CVR augmentation is accompanied by increasing, or more positive, BTR values.

In line with previous work examining hemispheric differences in the study of hemodynamics and brain temperature, ${ }^{36}$ we compared BTR and baseline brain temperature across hemispheres. Our analysis, however, did not reveal differences in baseline brain temperature and BTR between the ipsilateral and contralateral hemispheres such as those previously observed following isch- emic injury. ${ }^{16,36}$ However, the lack of hemispheric temperature differences is not surprising because even in cases of unilateral disease, consequential hemodynamic impairment may not be limited to the affected hemisphere and compromised function and tissue damage may exist bilaterally and heterogeneously. ${ }^{3}$ Consequently, temperature differences resulting from impaired cerebral perfusion and impaired autoregulation may be averaged out in coarse hemispheric comparisons. Nevertheless, the presence of significant random-effects differences in BTR and baseline brain temperature between hemispheres could suggest that in a larger sample size, a difference may exist.

We acknowledge several study limitations. While it was designed to test our hypothesis regarding the association between brain temperature changes and hemodynamic augmentation, the lengthy, multiparametric protocol necessitated a 2-day study, which limited the sample size. The findings nevertheless emphasize the feasibility of dynamic brain thermometry in this setting and compel more rigorous study of BTR as a prognostic biomarker. A challenge in the analysis was the observation of negative CVR values, suggesting the possibility of vascular steal and limitations in the measurement of CBF. ${ }^{10,29}$ As discussed above, several possible mechanisms may contribute to negative CVR values, particularly arterial transit artifacts, collateral rather than anterograde flow after ACZ, and the time scale of ASL-based measures of CVR. These may also contribute to spuriously high CVR values. As a result, continuous BOLD measurements may be a more reliable surrogate for the hemodynamic response to ACZ. The temporal dynamics of the ACZ response are worth considering. To mitigate potential errors related to a rapidly changing baseline, the initial BOLD signal used for $\mathrm{CVR}_{\mathrm{BOLD}}$ in our study was derived from a pre-ACZ phase of the examination spanning the initial 5 minutes of BOLD acquisition. A uniform protocol was prescribed in which a slow infusion of ACZ was then administered for 3-5 minutes without patient repositioning or scan interruption, followed by $\sim 10$ more minutes of uninterrupted BOLD signal acquisition. It was recently reported that $\mathrm{CVR}_{\mathrm{BOLD}}$ increases continuously following ACZ administration, reaching a plateau at approximately 8.5 minutes, which continues through the remainder of the acquisition. ${ }^{40}$ While CVR delays beyond 20 minutes are difficult to exclude, we expect that the window of maximal augmentation is likely captured within this study design.

While we cannot conclusively parcellate the competing hemodynamic effects from the technical challenges, further studies with MR-based methods to characterize functional activity, CBF, and metabolism in cerebrovascular disease would complement our results. While direct estimation of OEF and $\mathrm{CMRO}_{2}$ with MRbased techniques remains challenging, ongoing efforts to optimize MR oximetry measurements will enable further studies into the relationship of BTR with these metabolic parameters. ${ }^{8}$ A more thorough description could be facilitated through study of a healthy control population; however, administration of intravenous vasodilatory stimuli is not common practice in our experience. Other approaches such as induced hypercapnia or breathhold may be valuable in this regard, though the mechanism, depth, and magnitude of flow augmentation may differ. Last, the relatively small sample size and limited longitudinal follow-up information in this cohort of patients limit our characterization of 
BTR as a prognostic tool to predict future stroke risk in such patients. Further study in a larger and, ideally, prospectively collected cohort is warranted to better assess the prognostic power of BTR in a clinical population.

\section{CONCLUSIONS}

These findings suggest the feasibility and potential utility of noninvasive brain thermometry during the dynamic modulation of CBF and CVR, supporting further exploration of BTR as a potential biomarker in patients with steno-occlusive cerebrovascular disease. These results compel further study into the mechanistic nature of cerebral temperature regulation and the potential use of noninvasive MR thermometry in the diagnosis, prognostication, and treatment selection in chronic steno-occlusive disease.

Disclosures: Candace C. Fleischer-UNRELATED: Grants/Grants Pending: National Institutes of Health, Comments: National Institutes of Health postdoctoral fellowship (F32CA199834); Support for Travel to Meetings for the Study or Other Purposes: International Society for Magnetic Resonance in Medicine, Comments: International Society for Magnetic Resonance in Medicine travel award to attend the annual meeting in Singapore to present this work. Deqiang Qiu-UNRELATED: Grants/ Grants Pending: Medtronic and Siemens Healthcare, Comments: research support*. Fadi Nahab—UNRELATED: Expert Testimony: Grant Law Firm, Comments: pertains to cases of stroke treatment, not imaging of stroke; Payment for Lectures Including Service on Speakers Bureaus: Medtronic, American Heart Association/American Stroke Association, Genzyme, Comments: lectures on cryptogenic stroke, Fabry disease; Payment for Development of Educational Presentations: Imedex, Comments: education on cryptogenic stroke. Seena Dehkharghani-UNRELATED: Travel/Accommodations/Meeting Expenses Unrelated to Activities Listed: Society of Vascular and Interventional Neurology and All India Institute of Medical Sciences, Comments: travel expenses paid by the Society of Vascular and Interventional Neurology and All India Institute of Medical Sciences as an invited speaker. *Money paid to the institution.

\section{REFERENCES}

1. Yamauchi $\mathrm{H}$, Higashi $\mathrm{T}$, Kagawa $\mathrm{S}$, et al. Is misery perfusion still a predictor of stroke in symptomatic major cerebral artery disease? Brain 2012;135:2515-26 CrossRef Medline

2. Powers WJ. Stroke: misery perfusion in cerebrovascular disease-is it important? Nat Rev Neurol 2012;8:479-80 CrossRef Medline

3. Derdeyn CP, Videen TO, Yundt KD, et al. Variability of cerebral blood volume and oxygen extraction: stages of cerebral haemodynamic impairment revisited. Brain 2002;125(pt 3):595-607 Medline

4. Powers WJ. Cerebral hemodynamics in ischemic cerebrovascular disease. Ann Neurol 1991;29:231-40 CrossRef Medline

5. Nemoto EM, Yonas H, Pindzola RR, et al. PET OEF reactivity for hemodynamic compromise in occlusive vascular disease. J Neuroimaging 2007;17:54-60 CrossRef Medline

6. Heiss WD, Podreka I. Role of PET and SPECT in the assessment of ischemic cerebrovascular disease. Cerebrovasc Brain Metab Rev 1993;5:235-63 Medline

7. Okazawa $\mathrm{H}$, Yamauchi $\mathrm{H}$, Toyoda $\mathrm{H}$, et al. Relationship between vasodilatation and cerebral blood flow increase in impaired hemodynamics: a PET study with the acetazolamide test in cerebrovascular disease. J Nucl Med 2003;44:1875-83 Medline

8. Christen T, Bolar DS, Zaharchuk G. Imaging brain oxygenation with MRI using blood oxygenation approaches: methods, validation, and clinical applications. AJNR Am J Neuroradiol 2013;34:1113-23 CrossRef Medline

9. Mandell DM, Han JS, Poublanc J, et al. Mapping cerebrovascular reactivity using blood oxygen level-dependent MRI in patients with arterial steno-occlusive disease: comparison with arterial spin labeling MRI. Stroke 2008;39:2021-28 CrossRef Medline

10. Rogg J, Rutigliano $\mathrm{M}$, Yonas $\mathrm{H}$, et al. The acetazolamide challenge: imaging techniques designed to evaluate cerebral blood flow reserve. AJR Am J Roentgenol 1989;153:605-12 CrossRef Medline

11. Markus $H$, Cullinane M. Severely impaired cerebrovascular reactiv- ity predicts stroke and TIA risk in patients with carotid artery stenosis and occlusion. Brain 2001;124:457-67 CrossRef Medline

12. Gupta A, Chazen JL, Hartman M, et al. Cerebrovascular reserve and stroke risk in patients with carotid stenosis or occlusion: a systematic review and meta-analysis. Stroke 2012;43:2884-91 CrossRef Medline

13. Wang H, Wang B, Normoyle KP, et al. Brain temperature and its fundamental properties: a review for clinical neuroscientists. Front Neurosci 2014;8:307 CrossRef Medline

14. Sukstanskii AL, Yablonskiy DA. An analytical model of temperature regulation in human head. J Therm Biol 2004;29:583-87 CrossRef

15. Reith J, Jørgensen HS, Pedersen PM, et al. Body temperature in acute stroke: relation to stroke severity, infarct size, mortality, and outcome. Lancet 1996;347:422-25 CrossRef Medline

16. Karaszewski B, Wardlaw JM, Marshall I, et al. Measurement of brain temperature with magnetic resonance spectroscopy in acute ischemic stroke. Ann Neurol 2006;60:438-46 CrossRef Medline

17. Campos F, Blanco M, Barral D, et al. Influence of temperature on ischemic brain: basic and clinical principles. Neurochem Int 2012; 60:495-505 CrossRef Medline

18. Dehkharghani S, Bowen M, Haussen DC, et al. Body temperature modulates infarction growth following endovascular reperfusion. AJNR Am J Neuroradiol 2017;38:46-51 CrossRef Medline

19. Dehkharghani S, Fleischer CC, Qiu D, et al. Cerebral temperature dysregulation: MR thermographic monitoring in a nonhuman primate study of acute ischemic stroke. AJNR Am J Neuroradiol 2017; 38:712-20 CrossRef Medline

20. McDannold N. Quantitative MRI-based temperature mapping based on the proton resonant frequency shift: review of validation studies. Int J Hyperthermia 2005;21:533-46 CrossRef Medline

21. Corbett RJT, Purdy PD, Laptook AR, et al. Noninvasive measurement of brain temperature after stroke. AJNR Am J Neuroradiol 1999;20:1851-57 Medline

22. Castillo J, Martinez F, Leira R, et al. Mortality and morbidity of acute cerebral infarction related to temperature and basal analytic parameters. Cerebrovasc Dis 1994;4:66-71 CrossRef

23. Scheenen TW, Klomp DW, Wijnen JP, et al. Short echo time 1HMRSI of the human brain at $3 \mathrm{~T}$ with minimal chemical shift displacement errors using adiabatic refocusing pulses. Magn Reson Imaging 2008;59:1-6 Medline

24. Fleischer CC, Qiu D, Zhong X, et al. Multivoxel proton magnetic resonance spectroscopy for non-invasive thermometry: improvements in spectral quality using semiLASER with GRE shim. Proc Int Soc Magn Reson Med 2015;23:4062

25. Dehkharghani S, Fleischer CC, Qiu D, et al. Relationship between cerebrovascular reserve and brain temperature following acetazolamide challenge in patients with chronic steno-occlusive disease. Proc Int Soc Magn Reson Med 2016;24:0601

26. Chappell MA, MacIntosh BJ, Donahue MJ, et al. Separation of macrovascular signal in multi-inversion time arterial spin labelling MRI. Magn Reson Med 2010;63:1357-65 CrossRef Medline

27. Günther M, Oshio K, Feinberg DA. Single-shot 3D imaging techniques improve arterial spin labeling perfusion measurements. Magn Reson Med 2005;54:491-98 CrossRef Medline

28. Bonte FJ, Devous MD, Reisch JS, et al. The effect of acetazolamide on regional cerebral blood flow in patients with Alzheimer's disease or stroke as measured by single-photon emission computed tomography. Invest Radiol 1989;24:99-103 CrossRef Medline

29. Vagal AS, Leach JL, Fernandez-Ulloa M, et al. The acetazolamide challenge: techniques and applications in the evaluation of chronic cerebral ischemia. AJNR Am J Neuroradiol 2009;30:876-84 Medline

30. Okazawa $H$, Yamauchi $H$, Sugimoto K, et al. Effects of acetazolamide on cerebral blood flow, blood volume, and oxygen metabolism: a positron emission tomography study with healthy volunteers. J Cereb Blood Flow Metab 2001;21:1472-79 CrossRef Medline

31. Smith SM. Fast robust automated brain extraction. Hum Brain Mapp 2002;17:143-55 CrossRef Medline

32. Provencher SW. Automatic quantitation of localized in vivo $\mathbf{1 H}$ 
spectra with LCModel. NMR Biomed 2001;14:260-64 CrossRef Medline

33. Dehkharghani S, Mao H, Howell L, et al. Proton resonance frequency chemical shift thermometry: experimental design and validation toward high-resolution noninvasive temperature monitoring and in vivo experience in a nonhuman primate model of acute ischemic stroke. AJNR Am J Neuroradiol 2015;36:1128-35 CrossRef Medline

34. Kuroda K. Non-invasive MR thermography using the water proton chemical shift. Int J Hyperthermia 2005;21:547-60 CrossRef Medline

35. Kass RE, Raftery AE. Bayes factors. J Am Stat Assoc 1995;90:773-95 CrossRef

36. Ishigaki $\mathrm{D}$, Ogasawara $\mathrm{K}$, Yoshioka $\mathrm{Y}$, et al. Brain temperature measured using proton MR spectroscopy detects cerebral hemodynamic impairment in patients with unilateral chronic major cerebral artery steno-occlusive disease: comparison with positron emission tomography. Stroke 2009;40:3012-16 CrossRef Medline

37. Marshall I, Karaszewski B, Wardlaw JM, et al. Measurement of re- gional brain temperature using proton spectroscopic imaging: validation and application to acute ischemic stroke. Magn Reson Imaging 2006;24:699-706 CrossRef Medline

38. Murakami T, Ogasawara K, Yoshioka Y, et al. Brain temperature measured by using proton MR spectroscopy predicts cerebral hyperperfusion after carotid endarterectomy. Radiology 2010;256: 924-31 CrossRef Medline

39. Wu J, Dehkharghani S, Nahab F, et al. The effects of acetazolamide on the evaluation of cerebral hemodynamics and functional connectivity using blood oxygen level-dependent MR imaging in patients with chronic steno-occlusive disease of the anterior circulation. AJNR Am J Neuroradiol 2017;38:139-45 CrossRef Medline

40. Wu J, Dehkharghani S, Nahab F, et al. Acetazolamide-augmented dynamic bold (aczBOLD) imaging for assessing cerebrovascular reactivity in chronic steno-occlusive disease of the anterior circulation: an initial experience. Neuroimage Clin 2017;13:116-22 CrossRef Medline 